\title{
Obstructive Nephropathy
}

National Cancer Institute

\section{Source}

National Cancer Institute. Obstructive Nephropathy. NCI Thesaurus. Code C120902.

Renal damage and impaired renal function secondary to urinary tract obstruction. 